\title{
Related Factors of Suicidal Ideation among North Korean Refugee Youth in South Korea
}

\author{
Subin Park ${ }^{1}$ (D), Soo Jung Rim ${ }^{1}$ and Jin Yong Jun ${ }^{2, *}$ \\ 1 Department of Research Planning, Mental Health Research Institute, National Center for Mental Health, \\ Seoul 04933, Korea; subin-21@hanmail.net (S.P.); soojung0411@gmail.com (S.J.R.) \\ 2 Department of Psychiatry, National Center for Mental Health, Seoul 04933, Korea \\ * Correspondence: jjy826@naver.com; Tel.: +822-2204-0151
}

Received: 28 June 2018; Accepted: 7 August 2018; Published: 9 August 2018

\begin{abstract}
This study investigated the factors associated with suicidal ideation among 174 North Korean refugees (aged 13-27 years) residing in South Korea. Specifically, we compared sociodemographic, familial, social, and psychological characteristics between participants with and without suicidal ideation. Twenty-nine refugees (16.7\%) had exhibited suicidal ideation in the past 12 months. These refugees had significantly lower levels of familial cohesion $(U=1459.0 ; p<0.001)$, self-esteem $(U=1032.0 ; p<0.001)$, and resilience $(U=1190.0 ; p<0.001)$, as well as higher levels of expressional suppression $(U=1202.5 ; p<0.001)$ and post-traumatic stress disorder symptoms $(U=1303.0 ; p=0.001)$, (with Cohen's $d>0.5)$, compared to those without suicidal ideation. A multiple logistic regression analysis showed that the level of emotional suppression and familial cohesion were significantly associated with suicidal ideation, after controlling for the other variables. Familial and individual interventions, particularly those focused on encouraging emotional expression and familial cohesion, will be useful for North Korean refugee youth, who have a high risk of suicide.
\end{abstract}

Keywords: refugee; adolescent; risk factor; protective factor

\section{Introduction}

Almost half of the individuals who emigrate for reasons such as armed conflict, persecution, and economic pressure in their home countries are children and adolescents [1]. These children and youth not only suffer during their escape or displacement from their home country, but also after their arrival and during settlement in their new country [2]. Accordingly, researchers have been focusing greater attention on how to support their development following this rapid transition.

The number of North Korean refugees (NKRs) that settled in South Korea exceeded 30,000 in 2016, and around $40 \%$ of these were individuals aged 10-29 years [3]. Like other refugees, NKRs are exposed to traumatic events not only when residing in North Korea, but also during their escape [4]. Even after they have settled in South Korea, NKRs often struggle to adapt to their new culture [5]. NKR youths, in particular, face obstacles such as gaps in physical health, compared to same-age peers, perceived discrimination, culture shock, and low social support, all of which can lead to the high drop-out rates from school and unemployment after settling in South Korea [6,7]. Correspondingly, NKR youths often suffer from anxiety, depression, and post-traumatic stress disorder (PTSD) [8-10], all of which are associated with suicide ideation and attempts [11,12].

In 2009, suicide was reported as the leading cause of death among youth (i.e., 15-19 years old) in South Korea [13,14]. Since NKR youths are exposed to traumatic events and suffer in adapting to their new environment, they naturally require more clinical attention [15]; in fact, NKR youths appear to have a much higher prevalence of suicidal ideation compared to South Korean youths [16]. Since suicidal ideation is considered to have a strong association with suicidal behavior [17], it is 
important to investigate the factors related to suicidal ideation to prevent suicide. Klonsky and May [18], proposed the Three Step Theory of Suicide. The first step states that for an individual to have suicidal ideation, pain and feeling of hopelessness are needed. Then, if an individual has a suicidal ideation and also feels unconnected to life, he or she will move on to the next step: strong ideation. Finally, if an individual is capable of making a suicide attempt, he or she will proceed to the final step, actual attempt. According to this theory, suicide happens in a stepwise manner, so finding factors that are related to suicidal ideation is important to prevent individuals from proceeding to the next step. Since NKR youths experience traumatic events, the factors that are related to suicidal ideation of this group could be different from the general public. Therefore, exploring which factors are associated with this vulnerable group's suicidal ideation is important. However, to our knowledge, there has been no study investigating the factors influencing the suicidal ideation of this vulnerable group.

This study investigated the factors associated with suicidal ideation among NKR youths to prevent them from proceeding to the next step of the Three Step Theory. When exploring factors related to suicidal ideation of NKR youths, we have referred to a conceptual framework used by Reed and colleagues [1], which is based on the ecological model by Bronfenbrenner [19]. This conceptual framework [1] is used to explain the risk and protective factors for mental health of refugees. In this framework, the protective and risk factors of mental health are divided into four levels: individual (e.g., physical, psychological, or developmental disorders, age, sex, and exposure to violence), family (e.g., family composition, bereavement, and functioning), community (community social support), and societal (for example, language or cultural differences). We concentrated on factors from individual, family, and community level that could influence NKR youth's mental health. Factors included in the societal level were not included in the present study, due to aspects of data availability. Specifically, we explored sociodemographic (individual level), psychological (individual level), familial (family level), and social (community level) factors that might influence suicidal ideation of NKR youths in South Korea.

\section{Materials and Methods}

\subsection{Participants and Procedure}

We recruited NKR youths between 2017 and 2018 from two alternative schools for NKRs who are preparing for qualification examinations for middle- or high-school graduation. NKR youths who settle in South Korea go through a mental health screening program, of which this study was a part. All individuals who participated in the program were selected for recruitment, and consequently, a total of 174 NKR youths aged 13-27 years participated in this study. Participants completed a self-report questionnaire on their sociodemographic, familial, social, and psychological characteristics, as well as whether they had ever seriously considered suicide in the last 12 months. Informed consent was obtained from all participants. Among 174 participants, 29 (16.7\%) answered that they had seriously thought about suicide in the past 12 months. The study was reviewed and approved by the institutional review board of the National Center for Mental Health (No. 116271-2017-11).

\subsection{Measurements}

\subsubsection{Sociodemographic Characteristics}

Information on sociodemographic characteristics (i.e., age, sex, birthplace, parental educational levels, and type of residence), which is part of the individual level, was obtained.

\subsubsection{Familial and Social Support}

Familial (family level) and social support (community level) were assessed using the FACES III questionnaire [20,21], which comprises 20 items. There are two major parameters of family functioning that FACES III explores: cohesion and adaptability. Cohesion is assessed via statements such as "Family 
members know each other's close friends" and "Our family does things together". Adaptability is assessed via items such as "When problems arise we compromise" and "Family members say what they want". Each item is rated on a scale ranging from 1 ("almost never") to 5 ("almost always").

We also assessed participants' level of psychological support from others by asking participants "How much psychological support do you currently receive from your family, relatives, friends, and others around you?" Practical support was assessed by asking participants "How much practical support do you currently receive from your family, relatives, friends, and others around you?" For both questions, the responses were given on a 10-point Likert scale ( $1=$ "not at all"; $10=$ "receive enough support").

\subsubsection{Psychological Characteristics}

Psychological characteristics (i.e., resilience, self-esteem, cognitive style, impulsivity, and PTSD symptoms), which are included in the individual level, were assessed. We utilized the Brief Resilience Scale [22] to measure resilience (defined as the self-perceived ability to bounce back from stress). This scale contains three positive items (i.e., items 1,3, and 5) and three negative items (i.e., items 2, 4 , and 6). Each item is rated on a five-point scale ( $1=$ "strongly disagree"; $5=$ "strongly agree"). Negative items were reverse-scored. The total score ranges from 6 to 30, with higher scores indicating higher resilience.

The Rosenberg Self-Esteem Scale [23] was utilized to measure global self-worth. This scale contains ten items, and each rated on a 5 -point Likert scale $(1=$ "strongly disagree" to $5=$ "strongly agree"). The score ranges from 10 to 50, with higher scores indicating higher self-esteem.

The Emotion Regulation Questionnaire (ERQ) $[24,25]$ was utilized to assess cognitive reappraisal (e.g., "When I'm faced with a stressful situation, I make myself think about it in a way that helps me stay calm") and expressive suppression (e.g., "I control my emotions by not expressing them"), which are two emotion regulation strategies. Each item was rated on a 7-point Likert scale ( $1=$ "strongly disagree"; 7 = "strongly agree"). Higher scores on each subscale indicate the respondent's greater use of the corresponding emotion regulation strategy.

The Barratt Impulsivity Scale-Brief (BIS-Brief) was used to assess impulsivity [26]. This scale utilizes 8 items from the Korean version of the BIS-11, which has been evaluated for its validity and reliability [27]. Each item is rated on a 4-point Likert scale. Higher scores indicate higher impulsivity.

The Children's Revised Impact of Event Scale (CRIES) [28] was used to assess the degree of PTSD symptoms. The CRIES is a 13-item self-report scale adapted from the Impact of Event Scale [29]. Each item is scored on a four-point Likert scale $(0=$ "not at all"; $3=$ "often"). Higher scores indicate higher PTSD symptoms.

\subsubsection{Suicidal Ideation}

Suicidal ideation was measured with a single question asking participants "Have you ever seriously considered suicide in the last 12 months?" Participants who stated that they had a suicidal ideation in the last 12 months were classified as high risk group and were consulted by a physician if needed.

\subsection{Statistical Analyses}

We compared the sociodemographic characteristics of individuals with and without suicidal ideation using the independent $t$-test for continuous variables and chi-square test for categorical variables. Then, we investigated the difference between familial, social, and psychological characteristics of those with and without suicidal ideation. According to the Kolmogorov-Smirnov normality test, none of these variables had a normal distribution. Therefore, the Mann-Whitney U test was performed. Also, the effect size for each factor was retrieved, and those with medium size effect (Cohen's $d>0.5$ ) were accepted [30]. We then conducted a multiple binary logistic regression analysis with suicidal ideation as the main outcome variable, and the variables that significantly differed 
between suicidal and non-suicidal participants as the principal predictors. All statistical analyses were performed using SPSS Statistics 21.0 (IBM Corp, Armonk, NY, USA). Statistical significance was defined as an alpha of less than 0.05 .

\section{Results}

Table 1 shows the sociodemographic characteristics of the NKR youths with and without suicidal ideation. We observed no significant group differences in terms of sex, age, parental origin, parental educational levels, or type of residence.

Table 2 shows the familial, social, and psychological characteristics of NKR youths with and without suicidal ideation. NKR youths with suicidal ideation reported lower familial cohesion compared to those without suicidal ideation. The former group also had lower resilience and self-esteem, higher PTSD symptoms, and used emotional suppression more frequently compared to the latter group. There was a difference between familial adaptability between NKR youths with and without suicidal ideation, however, the effect was small.

Table 1. Sociodemographic characteristics of North Korean refugee youths with and without suicidal ideation.

\begin{tabular}{|c|c|c|c|c|}
\hline \multirow{2}{*}{ Characteristics } & $\begin{array}{l}\text { Without Suicidal } \\
\text { Ideation }(N=145)\end{array}$ & $\begin{array}{c}\text { With Suicidal } \\
\text { Ideation }(N=29)\end{array}$ & \multirow{2}{*}{$\chi^{2} / t$} & \multirow[t]{2}{*}{$p$} \\
\hline & $N(\%)$ & $N(\%)$ & & \\
\hline Sex, male & $46(31.7)$ & $11(37.9)$ & 0.42 & 0.516 \\
\hline Age, mean (SD) & $18.86(2.86)$ & $18.97(3.41)$ & 0.17 & 0.864 \\
\hline Birthplace & & & 1.34 & 0.247 \\
\hline North Korea & $82(56.6)$ & $13(44.8)$ & & \\
\hline China & $63(43.4)$ & $16(55.2)$ & & \\
\hline Paternal educational level & & & 0.04 & 0.851 \\
\hline High school degree or lower & $97(74.6)$ & $16(72.7)$ & & \\
\hline College degree or higher & $33(25.4)$ & $6(27.3)$ & & \\
\hline Maternal educational level & & & 1.68 & 0.195 \\
\hline High school degree or lower & $102(73.9)$ & $12(60.0)$ & & \\
\hline College degree or higher & $36(26.1)$ & $8(40.0)$ & & \\
\hline Residence & & & 0.10 & 0.752 \\
\hline With family & $56(38.9)$ & $10(35.7)$ & & \\
\hline With relatives/friends/alone or in a facility & $88(61.1)$ & $18(64.3)$ & & \\
\hline
\end{tabular}

Table 2. Familial, social, and psychological characteristics of North Korean refugee youths with and without suicidal ideation.

\begin{tabular}{|c|c|c|c|c|c|}
\hline \multirow{2}{*}{ Characteristics } & $\begin{array}{l}\text { Without Suicidal } \\
\text { Ideation }(N=145)\end{array}$ & $\begin{array}{c}\text { With Suicidal } \\
\text { Ideation }(N=29)\end{array}$ & \multirow{2}{*}{ Mann-Whitney U } & \multirow{2}{*}{$p$} & \multirow{2}{*}{ Cohen's $d$} \\
\hline & Mean Rank & Mean Rank & & & \\
\hline \multicolumn{6}{|l|}{ Familial } \\
\hline Familial adaptability & 91.94 & 65.31 & 1459.0 & 0.009 & 0.40 \\
\hline Familial cohesion & 93.51 & 57.47 & 1231.5 & $<0.001$ & 0.55 \\
\hline \multicolumn{6}{|l|}{ Social } \\
\hline Psychological support & 90.28 & 73.62 & 1700.0 & 0.100 & 0.25 \\
\hline Practical support & 90.25 & 73.76 & 1704.0 & 0.105 & 0.25 \\
\hline \multicolumn{6}{|l|}{ Psychological } \\
\hline Resilience & 93.79 & 56.03 & 1190.0 & $<0.001$ & 0.58 \\
\hline Self-esteem & 94.88 & 50.60 & 1032.0 & $<0.001$ & 0.69 \\
\hline Cognitive reappraisal & 90.29 & 73.57 & 1698.5 & 0.100 & 0.25 \\
\hline Emotional suppression & 81.29 & 118.53 & 1202.5 & $<0.001$ & 0.57 \\
\hline Impulsivity & 85.86 & 95.71 & 1864.5 & 0.334 & 0.15 \\
\hline PTSD symptoms & 81.55 & 114.07 & 1303.0 & 0.001 & 0.51 \\
\hline
\end{tabular}

Abbreviations: PTSD, post-traumatic stress disorder. 
Table 3 shows the results of the multiple regression analysis to identify the independent predictors of suicidal ideation. Only those factors with Cohen's $d>0.5$ and $p<0.05$ from Table 2 were included in the analysis. Emotional suppression and familial cohesion were significantly associated with suicidal ideation in this model.

Table 3. Variables associated with suicidal ideation in North Korean refugee youths.

\begin{tabular}{lcc}
\hline \multicolumn{1}{c}{ Characteristics } & AOR (95\% CI) & $p$ \\
\hline Familial cohesion & $0.94(0.89-0.99)$ & 0.039 \\
Resilience & $0.88(0.77-1.00)$ & 0.053 \\
Self-esteem & $0.93(0.84-1.02)$ & 0.111 \\
Emotional suppression & $1.32(1.08-1.60)$ & 0.006 \\
PTSD symptoms & $1.02(0.99-1.06)$ & 0.183 \\
\hline
\end{tabular}

Abbreviations: PTSD, post-traumatic stress disorder; AOR, adjusted odds ratio.

\section{Discussion}

This study explored the factors from the individual, family, and community level that are related to suicidal ideation of NKR youths. We found that NKR youths with suicidal ideation have lower level of familial cohesion, self-esteem, and resilience, with higher emotional suppression and PTSD symptoms than those without suicidal ideation. Moreover, according to the multiple regression analysis, emotional suppression and familial cohesion were associated with suicidal ideation. This shows that factors from individual and family level are associated with suicidal ideation of NKR youths.

In this study, $16.7 \%$ of the NKR youths reported having suicidal ideation in the past 12 months. According to our data, there were no significant differences in sociodemographic variables between youths with and without suicidal ideation. Past review studies [2,8] have found mixed results with regard to the associations between sociodemographic variables (e.g., sex, age, parental educational level, and residence) and the mental health of refugee youths. Obtaining a clear result is difficult, given the need to consider multiple potential confounders when investigating this association [2]. For instance, when investigating the association between age and mental health, confounders such as age at migration, age-related policies for education, age of first exposure to traumatic events, and many other factors must be considered.

As for familial factors, adolescents with suicidal ideation had significantly lower familial cohesion compared to those without suicidal ideation. Along with this result, multiple logistic regression analysis showed that familial cohesion was associated with lower odds of suicidal ideation, after controlling for other variables. The association between family cohesion and suicidal ideation might be explained by how family is a main source of emotional support [31], and the fact that family cohesion acts as a protective factor for mental problems (i.e., depressive symptoms) among NKRs [32]. NKR youths lose social connections by escaping from their country and settling to a new place. Accordingly, enhancing family cohesion might help adolescents endure traumatic experiences as well as adjust to their new environment, which in turn reduces their likelihood of suicide ideation. Interventions to help NKR youths to settle in Korea should include ways to build stronger familial cohesion.

As for the results concerning PTSD symptoms, these are in line with a case-control study [33] showing that NKRs tend to have more severe PTSD symptoms, on average, compared to South Koreans, and PTSD is known to be associated with suicide [11]. This finding highlights the importance of providing interventions aimed at preventing NKRs from developing PTSD symptoms after their exposure to traumatic events.

After arriving in South Korea, NKR youths must face another obstacle: acculturative stress. Acculturative stress manifests as homesickness, a sense of alienation, culture shock, feelings of marginalization, perceived discrimination, gaps in physical health, and problems in adjusting to their new education system [6,7], all of which lower NKRs' self-efficacy and self-esteem [6,33,34]. Moreover, 
various studies [6,34-37] have found that acculturative stress predicts psychiatric problems, including depression, anxiety, and PTSD. However, resilience might work as a buffer against these mental health problems-in other words, individual differences in resilience might help NKRs in dealing with environmental stress [8]. For instance, in one study [6], acculturative stress was related to greater depression and anxiety symptoms, while ego resiliency acted as a mediator in these relationships. Therefore, our findings that lower levels of self-esteem and resilience among NKRs exhibiting suicidal ideation are compatible with those of previous studies. This implies that programs that could improve NKR youths' self-efficacy and resilience need to be developed.

NKR youths with suicidal ideation exhibited lower emotional suppression compared to that of youths without suicidal ideation. Moreover, in the multiple regression analysis, emotional suppression was associated with higher odds of suicidal ideation of NKR youths. Alexithymia, defined as a difficulty in identifying, describing, and/or expressing emotions, has been suggested as a risk factor of psychiatric problems such as PTSD [38,39]. Indeed, Park, et al. [40] emphasized the important of expressing emotions among NKRs, stating that clearly identifying and expressing emotions alleviated PTSD symptoms among NKRs. Our result indicates that emotional suppression is not only associated with PTSD symptoms, but also suicidal ideation of NKR youths. This implies that helping NKR youths express their emotions after their traumatic experience is important for their mental health.

This study had several limitations. First, this study utilized a cross-sectional design. Therefore, we cannot infer any causal relationships between the studied variables and suicidal ideation among NKR youths. Next, the number of participants in our study was relatively small, with only 29 youths exhibiting suicidal ideation; this might influence the external validity of our results. For example, the small sample size might account for the negative results in the social support factor. Second, participants were students of only two schools, which mean that they were not likely drawn from a representative sample. Third, the data were collected through self-reports, and suicidal ideation was measured with a single question, which might have resulted in reporting bias [41]. Especially since suicidal ideation was measured with a single question, future studies need to use a validated scale to replicate our study. Fourth, we did not explore factors included in the societal level from the conceptual framework [1] we have referred to. Since NKR youths face various obstacles, including acculturative stress [6,7], factors included in the societal level need to be considered in future studies. Finally, the conceptual framework [1] that we have referred to was developed based on Bronfenbrenner's model [19]. Since we did not use the fundamental model, we may have missed some aspects from the model which could be associated with NKR youth's suicidal ideation.

Despite the aforementioned limitations, this study had several meaningful results. Specifically, NKR youths are at risk of suicide. To lower this risk, familial and individual interventions, particularly those focusing on emotional expression and familial cohesion, are essential. We believe that our results will help experts in related fields intervene in NKR youths' suicidal ideation. Further studies using a larger, more representative sample, and a longitudinal design, are needed to confirm our results. Additionally, future studies should aim to develop intervention programs for NKR youths and examine their effectiveness.

Author Contributions: Conceptualization, J.Y.J. and S.P.; Analysis, S.P.; Data collection, J.Y.J.; Writing-Original Draft Preparation, S.P.; Writing-Review and Editing, S.J.R.; Supervision, J.Y.J.; Funding Acquisition, S.P.

Funding: This work was supported by a National Research Foundation of Korea (NRF) grant funded by the Korean Government (NRF-2016R1D1A1B03931290).

Conflicts of Interest: The authors declare no conflict of interest. 


\section{References}

1. Reed, R.V.; Fazel, M.; Jones, L.; Panter-Brick, C.; Stein, A. Mental health of displaced and refugee children resettled in low-income and middle-income countries: Risk and protective factors. Lancet 2012, 379, 250-265. [CrossRef]

2. Fazel, M.; Reed, R.V.; Panter-Brick, C.; Stein, A. Mental health of displaced and refugee children resettled in high-income countries: Risk and protective factors. Lancet 2012, 379, 266-282. [CrossRef]

3. Ministry of Unification. Number of North Korean Defectors by Age Group. Available online: http:/ / www. unikorea.go.kr/eng-unikorea/relations/statistics/defectors/ (accessed on 3 January 2018).

4. Jeon, W.; Yu, S.; Cho, Y.; Eom, J. Traumatic experiences and mental health of North Korean refugees in South Korea. Psychiatry Investig. 2008, 5, 213-220. [CrossRef] [PubMed]

5. Jeon, W.; Min, S.; Lee, M.; Lee, E. A study on adaptation of North Koreans in South Korea. J. Korean Neuropsychiatr. Assoc. 1997, 36, 145-161.

6. Kim, Y.; Cho, Y.; Kim, H. A mediation effect of ego resiliency between stresses and mental health of North Korean refugee youth in South Korea. Child Adolesc. Soc. Work J. 2015, 32, 481-490. [CrossRef]

7. Sung, J.; Go, M. Resettling in South Korea: Challenges for youth North Korean Refugees; The Asan Institute for Policy Studies: Seoul, Korea, 2014; p. 18.

8. Lee, Y.; Lee, M.; Park, S. Mental health status of North Korean refugees in South Korea and risk and protective factors: A 10-year review of the literature. Eur. J. Psychotraumatol. 2017, 8, 1369833. [CrossRef] [PubMed]

9. Choi, S.K.; Min, S.J.; Cho, M.S.; Joung, H.; Park, S.M. Anxiety and depression among North Korean young defectors in South Korea and their association with health-related quality of life. Yonsei Med. J. 2011, 52, 502-509. [CrossRef] [PubMed]

10. Park, S.; Lee, M.; Jeon, J.Y. Factors affecting depressive symptoms among North Korean adolescent refugees residing in South Korea. Int. J. Environ. Res. Public Health 2017, 14, 912. [CrossRef] [PubMed]

11. Stevens, D.; Wilcox, H.C.; MacKinnon, D.F.; Mondimore, F.M.; Schweizer, B.; Jancic, D.; Coryell, W.H.; Weissman, M.M.; Levinson, D.F.; Potash, J.B. Posttraumatic stress disorder increases risk for suicide attempt in adults with recurrent major depression. Depress. Anxiety 2013, 30, 940-946. [CrossRef] [PubMed]

12. Sareen, J.; Cox, B.J.; Afifi, T.O.; de Graaf, R.; Asmundson, G.J.; ten Have, M.; Stein, M.B. Anxiety disorders and risk for suicidal ideation and suicide attempts: A population-based longitudinal study of adults. Arch. Gen. Psychiatry 2005, 62, 1249-1257. [CrossRef] [PubMed]

13. Statistics Korea. Suicide Rates: Aged from 15 to 19 Years in Korea; Statistics Korea: Daegeon, Korea, 2012.

14. Park, S. Brief report: Sex differences in suicide rates and suicide methods among adolescents in South Korea, Japan, Finland, and the U.S. J. Adolesc. 2015, 40, 74-77. [CrossRef] [PubMed]

15. Lee, Y.; Shin, O.; Lim, M. The psychological problems of North Korean adolescent refugees living in South Korea. Psychiatry Investig. 2012, 9, 217-222. [CrossRef] [PubMed]

16. Kim, M.J.; Yu, S.Y.; Kim, S.; Won, C.W.; Choi, H.; Kim, B.S. Health behavior and factors associated with depression in North Korean adolescent defectors in South Korea: The Korea Youth Risk Behavior Web-based Survey, 2011-2014. Korean J. Fam. Med. 2017, 38, 256-262. [CrossRef] [PubMed]

17. Lewinsohn, P.M.; Rohde, P.; Seeley, J.R. Adolescent suicidal ideation and attempts: Prevalence, risk factors, and clinical implications. Clin. Psychol. Sci. Pract. 1996, 3, 25-36. [CrossRef]

18. Klonsky, E.D.; May, A.M. The Three-Step Theory (3ST): A new theory of suicide rooted in the "Ideation-to-action" Framework. Int. J. Cogn. Ther. 2015, 8, 114-129. [CrossRef]

19. Bronfenbrenner, U. The Ecological of Human Development: Experiments by Nature and Design; Harvard University Press: Cambridge, MA, USA, 1979; pp. 16-44.

20. Yun, B.B.; Lee, H.L.; Kwak, K.W.; Oh, M.K.; Lim, J.H.; Lee, G.L. A study on reliability and validity of FACES 3. Korean J. Fam. Med. 1990, 11, 8-17.

21. Olson, D.H. Family Adaptation and Cohesion Scales; University of Minnesota: St. Paul, MN, USA, 1985.

22. Smith, B.W.; Dalen, J.; Wiggins, K.; Tooley, E.; Christopher, P.; Bernard, J. The brief resilience scale: Assessing the ability to bounce back. Int. J. Behav. Med. 2008, 15, 194-200. [CrossRef] [PubMed]

23. Lee, J.Y.; Nam, S.K.; Lee, M.K.; Lee, J.H.; Lee, S.M. The Rosenberg Self-esteem Scale: A validation study. Korean J. Couns. Psychother. 2009, 21, 73-89.

24. Gross, J.J.; John, O.P. Individual differences in two emotion regulation processes: Implications for affect, relationships, and well-being. J. Pers. Soc. Psychol. 2003, 85, 348-362. [CrossRef] [PubMed] 
25. Han, S.H.; Hyun, O.K. Relationships of positive and negative emotion to cognitive reappraisal and expressive suppression emotional regulation strategies and self-control in adolescence. Korean J. Child Stud. 2006, 27, $1-11$.

26. Steinberg, L.; Sharp, C.; Stanford, M.S.; Tharp, A.T. New tricks for an old measure: The development of the Barratt Impulsiveness Scale-Brief (BIS-Brief). Psychol. Assess. 2013, 25, 216-226. [CrossRef] [PubMed]

27. Heo, S.Y.; Oh, J.Y.; Kim, J.H. The Korean version of the Barratt Impulsiveness Scale, 11th version: Its reliability and validity. Korean J. Psychol. Gen. 2012, 31, 769-782.

28. Smith, P.; Perrin, S.; Dyregrov, A.; Yule, W. Principal components analysis of the impact of event scale with children in war. Pers. Indiv. Differ. 2003, 34, 315-322. [CrossRef]

29. Horowitz, M.; Wilner, N.; Alvarez, W. Impact of Event Scale: A measure of subjective stress. Psychosom. Med. 1979, 41, 209-218. [CrossRef] [PubMed]

30. Cohen, J. Statistical Power Analysis for the Behavioural Sciences, 2nd ed.; Earlbaum: Hillsdale, NJ, USA, 1988.

31. Schweitzer, R.; Greenslade, J.; Kagee, A. Coping and resilience in refugees from the Sudan: A narrative account. Aust. N. Z. J. Psychiatry 2007, 41, 282-288. [CrossRef] [PubMed]

32. Nam, B.; Kim, J.Y.; DeVylder, J.E.; Song, A. Family functioning, resilience, and depression among North Korean refugees. Psychiatry Res. 2016, 245, 451-457. [CrossRef] [PubMed]

33. Chang, M.; Son, E. Complex PTSD symptoms and psychological problems of the North Korean defectors. Korean J. Health Psychol. 2014, 19, 973-999. [CrossRef]

34. Lim, S.; Han, S. A predictive model on North Korean refugees' adaptation to South Korean society: Resilience in response to psychological trauma. Asian Nurs. Res. (Korean Soc. Nurs. Sci.) 2016, 10, 164-172. [CrossRef] [PubMed]

35. Kim, M.; Lee, D. Adaptation of North Korean adolescent refugees to South Korean society: A review of literature. J. Rehabil. Psychol. 2013, 20, 39-64. [CrossRef]

36. Kim, Y. Predictors for mental health problems among young North Korean refugees in South Korea. Contemp. Soc. Multicult. 2013, 3, 264-285.

37. Lee, K. A study on factors influencing on mental health in North Korean defector youth: The mediating effects of acculturative stress. Contemp. Soc. Multicult. 2011, 1, 157-180.

38. Bagby, R.M.; Parker, J.D.; Taylor, G.J. The twenty-item Toronto Alexithymia Scale-I. Item selection and cross-validation of the factor structure. J. Psychosom. Res. 1994, 38, 23-32. [CrossRef]

39. Bagby, R.M.; Taylor, G.J.; Parker, J.D. The twenty-item Toronto alexithymia scale-II. Convergent, discriminant, and concurrent validity. J. Psychosom. Res. 1994, 38, 33-40. [CrossRef]

40. Park, S.; Hatim Sulaiman, A.; Srisurapanont, M.; Chang, S.M.; Liu, C.Y.; Bautista, D.; Ge, L.; Choon Chua, H.; Pyo Hong, J.; Mood Disorders Research: Asian and Australian Network. The association of suicide risk with negative life events and social support according to gender in Asian patients with major depressive disorder. Psychiatry Res. 2015, 228, 277-282. [CrossRef] [PubMed]

41. Millner, A.J.; Lee, M.D.; Nock, M.K. Single-item measurement of suicidal behaviors: Validity and consequences of misclassification. PLoS ONE 2015, 10, e0141606. [CrossRef] [PubMed]

(C) 2018 by the authors. Licensee MDPI, Basel, Switzerland. This article is an open access article distributed under the terms and conditions of the Creative Commons Attribution (CC BY) license (http:/ / creativecommons.org/licenses/by/4.0/). 